\title{
Knowledge, Attitude and Practice of Community Towards Zoonotic Importance of Toxoplasma Infection in Central Afar Region, North East Ethiopia
}

\author{
Angesom Hadush Desta \\ College of Veterinary Medicine, Samara University, Samara, Ethiopia
}

Email address:

meryangieboy@gmail.com

\section{To cite this article:}

Angesom Hadush Desta. Knowledge, Attitude and Practice of Community Towards Zoonotic Importance of Toxoplasma Infection in Central Afar Region, North East Ethiopia. International Journal of Biomedical Science and Engineering. Vol. 3, No. 6, 2015, pp. 74-81. doi: 10.11648/j.ijbse.20150306.12

\begin{abstract}
Toxoplasmosis is one of the major global zoonotic diseases which have a great impact on human health with more serious outcomes in immunocompromised and pregnant women. A cross-sectional study was carried out to assess the knowledge, attitude and practice of the community towards zoonotic importance of the disease in central Afar region of Northeast Ethiopia using structured questionnaire, key informants interview and focal group discussion. According to the logistic regression analysis of pregnant women's awareness on zoonotic Toxoplasma infection, literacy was found statistically significant $(\mathrm{P}<0.05)$ in association with awareness of the pregnant women about the disease. According to the respondents' answers on the general knowledge of toxoplasmosis, majority of these pregnant women have no knowledge or not sure about this parasitic infection. Most of the respondent pregnant women were not sure about the risk factors, symptoms, and timing of infection of toxoplasmosis. However, they routinely practice primary preventive behaviors, particularly good hygienic condition that can prevent them from Toxoplasma infection but this is due to their native knowledge and thinking that ensuring general and personal hygiene can prevent all types of infection. During the study, 24 animal health assistants were participated and almost all had knowledge on zoonotic Toxoplasma infection, transmission, treatment, control and prevention. However, all of them had never diagnosed the disease in animals. Moreover, out of the 68 medical professionals included in the study, only $23(33.8 \%)$ had knowledge on zoonotic Toxoplasma infection which is lower than expected when compared with importance of the disease in human health particularly pregnant women and children. This study showed that there is knowledge gap about the disease and its zoonotic importance. Therefore, refreshing training to medical professionals including health extension workers, imparting health education in one health approach to the community at large and pregnant women in particular and strengthening the diagnostic facility and advising pregnant women to regularly check for toxoplasma infection is mandatory.
\end{abstract}

Keywords: Afar, Cat, Ethiopia, Pregnant Women, Public Health, Toxoplasmosis

\section{Introduction}

Toxoplasma gondii is an intracellular protozoan organism with large number of intermediate hosts, including all warmblooded animals and humans [1]. Felids, particularly the domestic cat, are its definitive host and the only animal species in which oocyst develop [2]. Because of its broad host range, its high infection rates and its benign co-existence with the host, $T$. gondii is regarded as one of the most successful parasite on the earth. Toxoplasma is a global parasite with no known geographic boundaries [3].

The transmission of Toxoplasma gondii to humans occurs through accidental ingestion of sporulated oocysts or the consumption of raw or undercooked meat. Among meat producing animals pigs, sheep and goats relatively often harbour Toxoplasma gondii cysts in edible tissues and, therefore, raw or undercooked meat from these animals constitutes a major risk to humans. In areas where goat milk is utilized, unpasteurized milk from acutely diseased goats is also an important source of infection especially to children. Toxoplasma gondii can also be transplacentally transmitted from the mother to the offspring if the infection is contracted during pregnancy [4]. Congenital toxoplasmosis is a hazard in areas where the disease is prevalent [5].

The infection in pregnant women may cause devastating 
effects in the fetus. If the infected tissue of an animal being consumed; it acts as a transmission mode of infection. Furthermore, this parasitic infection can be transmitted by ingestion of Toxoplasma oocysts, contaminated fruits and vegetables or unclean water, through blood transfusion, and by receiving organ transplant .Exposures to contaminated feces while cleaning cat's litter, gardening, or handling contaminated soils are some of the ways of this parasitic infection being transmitted[6].

All forms of toxoplasmosis that occur in normal individuals may also occur in higher degree in immuno compromised patients. People who have previously acquired toxoplasmosis, with or without manifestation of the disease may suffer a devastating relapse if their immune defenses, particularly cell-mediated immunity are impaired as in the case of Acquired Immuno Deficiency Syndrome-AIDS [5].

The importance of this parasite in food safety, human health and animal husbandry has been well recognized [7]. $T$. gondii cause Toxoplasmosis which is an important zoonotic disease and responsible for major economic losses in all classes of livestock through abortion, stillbirth and neonatal losses [8, 9-10].Approximately one-third of the human world's population is infected by $T$. gondii $[11,7]$.

Even though a number of investigations have been carried out in many countries of the world, little is known on the epidemiology and public health importance of toxoplasmosis in Ethiopia. Ethiopia hosts millions of felines (both domestic and wild cats), livestock, birds and wild animals with extensive type of production. These millions of animals are raised under various agro-ecological zones, however, only few studies have been carried out yet. Results of the previous studies have shown that prevalence of anti $T$. gondii antibodies ranged from $11.9 \%$ to $89.9 \%[12-17]$.

In this country particularly in the Afar region, the causes of most abortions, stillbirths and neonatal mortalities remain unexplored and the relationship with seroprevalence of toxoplasmosis has not been investigated. Besides, there is a habit of eating raw and / or under cooked meat, unavoidable contact between humans and domestic animals and high prevalence of HIV/AIDS. Cats have been associated with domestic animals as an aid to rodent control. The prevalence of Toxoplasmosis in different agro-ecological zone and the relative contribution of the various routes of transmission in humans have not been adequately studied. Knowledge of the community on symptoms, transmission methods, treatment, prevention and control of the disease is not assessed. The attitude and current practice towards zoonotic importance of the disease is not studied. Therefore, the general objective of this study was to assess the knowledge, attitude and practice of the community towards zoonotic importance of toxoplasmosis in central zone of Afar region, Ethiopia.

\section{Methods}

\subsection{Description of Study Area}

Afar regional state is located in the Great Rift Valley, comprising semi-arid rangeland in northeastern Ethiopia. According to regional estimates the livestock population of Afar is about 10.12 million TLU. The Afar Regional State has five administrative zones, which are further subdivided into 32 districts. Pastoralism and agro- pastoralism are the two major livelihood ways practiced in the region. The population of the region is estimated to be about 1.2 million of which $90 \%$ are pastoralists and 10\% agro-pastoral. The study was conducted in Asayita district of central zone of this region. This district has a total population of 50,803, of whom 27,284 are men and 23,519 women; with an area of 1,678.28 square kilometers, Asayita has a population density of 30.27 . While 16,052 or $31.60 \%$ are urban inhabitants, a further 9,358 or $18.42 \%$ are pastoralists. A total of 11,096 households were counted in this district, which results in an average of 4.6 persons to a household, and 11,404 housing units [18].

\subsection{Study Population}

The target groups for the interview were pregnant women, animal health and medical professionals found in Asayita district.

\subsection{Study Design}

A cross-sectional study design was conducted to assess knowledge, attitude and practice of the community towards zoonotic importance of the disease. The study was conducted in both urban and rural areas of Asayita district. Pastoralist association (PA) is the lowest administrative unit within the district that was considered during the study. Accordingly, four PAs (two urban and two rural) from the district were randomly selected.

\subsection{Sampling Methods}

Asayita district was selected due to easier accessibility and affordability. There are 13 PAs in Asayita district. About $30 \%$ of the PAs in the district were included in the study on the basis of feasibility and affordability. Hence, four PAs were selected randomly. The PAs are stratified in to urban and rural areas based on the settlement, accordingly, two PAs from urban and the other two from rural areas were included in the study. There is no previous year's report on the knowledge and practice of the community towards the infection in the study area but there is a report of $11 \%$ in Malaysia, Philippines and Thailand [19]. Therefore, the average expected response distribution is assumed to be $11 \%$ within $95 \%$ Confidence Interval (CI) at $5 \%$ desired accuracy. Hence, using the formula of:

$$
\mathrm{n}=\frac{1.96 \times \mathrm{RD} \times(1-\mathrm{RD})}{\mathrm{d}^{2}}
$$

Where $\mathrm{n}=$ sample size, $\mathrm{d}=$ desired absolute precision (0.05), $\mathrm{RD}=$ response distribution $(0.11)$, thus the desired sample size is $\mathrm{n} \approx 77$. However, to increase the accuracy of the study the sample size is increased to 156 . Since there is no well recorded data of the households having pregnant women in each PAs of the district, the total sample size was distributed equally (39 pregnant women each) to all selected 
PAs. All households having at least one pregnant woman were registered in each PAs with the help of health extension workers and PAs administrators before sampling. Then, 39 households were randomly selected from each PAs in which one pregnant woman interviewed from each household. In addition, 24 animal health assistants and 68 medical professionals including health extension workers were included based on their availability and willingness to participate in the study.

\subsection{Study Methodology}

A suitable tool was prepared to assess the knowledge, attitude and practice/KAP of the community settled on both rural and urban areas of the study areas. A structured questionnaire was administered through trained data collectors after pre- testing in the field and properly translated to the local language 'Afarigna'. Written informed consent was obtained from each of the respondents after the objective of the survey was explained to them before starting the interview. The questionnaire was designed to assess the knowledge, attitude and practice/KAP of the community settled on both rural and urban areas towards zoonotic importance of the disease. The KAP tool focused on knowledge and attitude of the community on causes, symptoms, transmission methods, and treatment, control and prevention mechanisms of toxoplasmosis. In addition, it focuses on animal products and vegetables handling and consumption behavior, cat litter handling methods, contact with soil, history of abortion and source of drinking water etc. Socio-demographic history of each respondent was also recorded. The target groups of the study were randomly selected pregnant women, animal health assistants in veterinary clinics, medical professionals in hospitals and health centers of the selected study areas.

\subsection{Data Management and Statistical Analysis}

The data were summarized, cleaned and compiled after coded data are stored in Microsoft Excel 2007 spread sheet and transferred to SPSS ${ }^{\circledR}$ Version 20 for statistical analysis. Descriptive and analytic statistics were computed using software SPSS ${ }^{\circ}$ Version 20. Logistic regression and Chisquare test $\left(X^{2}\right)$ were employed to see the association of demographic history with knowledge of the respondents. The degree of association were computed using Odds ratio (OR) and $95 \%$ confidence interval (CI).

\subsection{Ethical Approval}

All respondents were informed about the objective of the study and written informed consents were obtained from all respondents and confidentiality was assured.

\section{Results}

\subsection{KAP Survey on Pregnant Women}

Knowledge, attitude and practice of pregnant women towards zoonotic importance of the disease were assessed using a structured questionnaire. A total of 156 respondents (78 respondents each from urban and rural areas) were interviewed. Out of the 156 respondents, only $48(30.8 \%)$ were literate, $108(69.2 \%)$ were illiterate and again $42(26.9 \%)$ of them were single and 114(73.1\%) were married.

According to the logistic regression analysis, literacy was found statistically significant $(\mathrm{P}<0.05)$ in association with awareness of the pregnant women about the disease (Table 1)

Table 1. Logistic regression analysis of pregnant women's awareness on zoonotic Toxoplasma infection in Asayita district of Afar region, North east Ethiopia.

\begin{tabular}{|c|c|c|c|c|}
\hline \multirow{2}{*}{ Category } & \multirow{2}{*}{ № } & \multirow{2}{*}{ Awareness (\%) } & \multicolumn{2}{|l|}{ Univariate } \\
\hline & & & $\mathrm{OR}^{\mathrm{a}}\left(95 \% \mathrm{CI}^{\mathrm{b}}\right)$ & P-value \\
\hline \multicolumn{5}{|l|}{ Residence } \\
\hline Urban* & 78 & 8.97 & - & \\
\hline Rural & 78 & 2.56 & $2.74(0.30-5.05)$ & 0.37 \\
\hline \multicolumn{5}{|l|}{ Age } \\
\hline$\leq 20 *$ & 22 & 9.09 & - & \\
\hline $21-30$ & 56 & 8.93 & $4.00(1.66-9.63)$ & 0.27 \\
\hline $31-40$ & 64 & 3.12 & $1.87(1.40-2.48)$ & 0.36 \\
\hline$>40$ & 14 & 0 & $\begin{array}{l}6.27(2.31- \\
11.02)\end{array}$ & 0.11 \\
\hline \multicolumn{5}{|l|}{ Marital status } \\
\hline Single* & 42 & 9.52 & - & \\
\hline Married & 114 & 4.38 & $1.23(0.19-7.65)$ & 0.82 \\
\hline \multicolumn{5}{|l|}{ № of children } \\
\hline None* & 32 & 12.50 & - & \\
\hline 1 & 51 & 5.88 & $0.31(0.02-4.37)$ & 0.39 \\
\hline$>1$ & 73 & 2.74 & $0.71(0.09-5.40)$ & 0.74 \\
\hline \multicolumn{5}{|l|}{ Education } \\
\hline Literate* & 48 & 18.75 & - & \\
\hline Illiterate & 108 & 0 & $1.10(0.03-2.32)$ & 0.01 \\
\hline \multicolumn{5}{|l|}{$\begin{array}{l}\text { History of } \\
\text { abortion }\end{array}$} \\
\hline Yes* & 36 & 8.34 & - & \\
\hline No & 120 & 5.00 & $0.58(0.14-2.44)$ & 0.46 \\
\hline
\end{tabular}

a: Odds ratio; b: Confidence interval; *: Reference category.

\subsection{Knowledge, Attitude and Practice of Pregnant Women About Toxoplasmosis}

About $9(5.77 \%)$ of the respondent pregnant women have ever heard or read about toxoplasmosis and only $12(7.70 \%)$ have ever been tested for toxoplasmosis (Table 2).

Table 2. Knowledge, attitude and practice of pregnant women towards toxoplasmosis.

\begin{tabular}{|c|c|c|c|}
\hline \multirow{2}{*}{ Issues raised } & \multicolumn{3}{|c|}{ Response category $(\mathrm{N}=156)$} \\
\hline & Yes $(\%)$ & No $(\%)$ & Not sure $(\%)$ \\
\hline \multicolumn{4}{|l|}{ General knowledge } \\
\hline Have you ever heard or read about toxoplasmosis? & 5.77 & 85.89 & 8.34 \\
\hline Have you ever been tested for toxoplasmosis? & 7.70 & 76.92 & 15.38 \\
\hline Is toxoplasmosis caused by an infection? & 5.77 & 12.82 & 81.41 \\
\hline
\end{tabular}




\begin{tabular}{|c|c|c|c|}
\hline \multirow{2}{*}{ Issues raised } & \multicolumn{3}{|c|}{ Response category (N=156) } \\
\hline & Yes (\%) & No (\%) & Not sure $(\%)$ \\
\hline Is the parasite shed in the feces of infected cats? & 7.05 & 35.90 & 57.05 \\
\hline Is the parasite found in raw or undercooked meat? & 9.62 & 24.36 & 66.02 \\
\hline Is the parasite found in unpasteurized milk? & 7.69 & 35.90 & 56.41 \\
\hline \multicolumn{4}{|l|}{ Risk factors } \\
\hline Can people get toxoplasmosis by changing cat litter? & 7.69 & 41.67 & 50.64 \\
\hline Can people get toxoplasmosis by eating raw/undercooked meat? & 3.21 & 69.87 & 26.92 \\
\hline Can people get toxoplasmosis by drinking raw milk? & 7.06 & 51.92 & 41.02 \\
\hline Can people get toxoplasmosis by eating raw vegetables? & 81.41 & 3.85 & 14.74 \\
\hline Can people get toxoplasmosis by receiving blood transfusion? & 62.82 & 7.69 & 29.49 \\
\hline Can people get toxoplasmosis by drinking untreated water? & 89.74 & 1.92 & 8.34 \\
\hline Can people get toxoplasmosis by gardening without gloves? & 24.36 & 4.49 & 71.15 \\
\hline \multicolumn{4}{|l|}{ Symptoms and timing of infection } \\
\hline Can pregnant women develop serious complications after infection with toxoplasmosis ( $T$. gondii)? & 10.26 & 20.51 & 69.23 \\
\hline Can unborn and/or newborn children develop serious complications after infection with toxoplasmosis ( $T$. gondii)? & 37.82 & 5.13 & 57.05 \\
\hline Can toxoplasmosis in a pregnant woman cause fever and feeling like you have the 'flu'? & 47.44 & 7.05 & 45.51 \\
\hline Can toxoplasmosis in a pregnant woman cause swollen glands (lymph nodes)? & 16.02 & 5.13 & 78.85 \\
\hline Can toxoplasmosis in a pregnant woman cause no symptoms? & 6.41 & 76.28 & 17.31 \\
\hline $\begin{array}{l}\text { Toxoplasmosis }(T \text {. gondii) can only be passed from a pregnant woman to her fetus if she is newly infected during } \\
\text { that pregnancy }\end{array}$ & 3.20 & 11.54 & 85.26 \\
\hline $\begin{array}{l}\text { Toxoplasmosis ( } T \text {. gondii) is rarely passed from a pregnant woman to her fetus if she was infected before becoming } \\
\text { pregnant }\end{array}$ & 19.23 & 16.67 & 64.10 \\
\hline A baby with toxoplasmosis may have no signs of illness at birth, but develop illness later & 34.62 & 10.89 & 54.49 \\
\hline A baby with toxoplasmosis may have vision problems & 44.23 & 17.31 & 38.46 \\
\hline A baby with toxoplasmosis may be treated with medicine & 90.38 & 2.56 & 7.06 \\
\hline \multicolumn{4}{|l|}{ Prevention knowledge } \\
\hline Feeding your cat dry or commercial cat food and not letting it kill and eat rodents & 10.25 & 4.49 & 85.26 \\
\hline Avoiding stray cats & 90.38 & 5.13 & 4.49 \\
\hline Letting someone else change the cat's litter box & 51.28 & 10.26 & 38.46 \\
\hline Making sure the cat's litter box is changed daily & 63.46 & 3.85 & 32.69 \\
\hline Toxoplasmosis can be prevented by cooking meat well until no pink is seen and the juices run clear & 75.64 & 8.34 & 16.02 \\
\hline Toxoplasmosis can be prevented by thoroughly washing and/or peeling all fruits and vegetables before eating them & 85.26 & 5.13 & 9.61 \\
\hline Toxoplasmosis may be prevented by cleaning all cutting boards and utensils thoroughly after each use & 95.51 & 1.92 & 2.57 \\
\hline \multicolumn{4}{|l|}{ Preventive behaviour } \\
\hline Do you routinely wash your hands after gardening? & 77.56 & 19.87 & 2.57 \\
\hline Do you routinely wash your hands after changing cat litter? & 64.74 & 26.28 & 8.98 \\
\hline Do you routinely wash your hands after handling raw meat? & 62.18 & 32.69 & 5.13 \\
\hline Do you cook meat well before eating? & 84.61 & 8.34 & 7.05 \\
\hline Do you avoid drinking raw milk? & 6.41 & 89.74 & 3.85 \\
\hline Do you avoid drinking untreated water? & 8.97 & 85.90 & 5.13 \\
\hline
\end{tabular}

\subsection{Questionnaire Survey on Animal Health Assistants and Medical Professionals}

A separate questionnaire was administered to animal health assistants and medical professionals to evaluate the awareness and practices of the professionals on zoonotic Toxoplasma infection. During the study, 24 animal health assistants were participated and almost all had knowledge on zoonotic Toxoplasma infection, transmission, treatment, control and prevention. However, all of them had never diagnosed the disease in animals.
Moreover, out of the 68 medical professionals included in the study, only $23(33.82 \%)$ had knowledge on zoonotic Toxoplasma infection. Majority of the professionals who had knowledge on the disease knew the transmission, treatment, control and prevention methods correctly. But none of them had ever diagnosed the disease in humans and according to the respondents, the reason for the diagnosis problem was lack of facility $(92.65 \%)$ and no attention was given to the disease (89.71\%) (Table 3).

Table 3. Awareness and practices of medical professionals about zoonotic Toxoplasma infection.

\begin{tabular}{lll}
\hline Questionnaire items & Response category & Respondent\% (N=68) \\
\hline Knowledge about zoonotic toxoplasmosis & Yes & 33.82 \\
& No & 66.18 \\
& Contact with cat feces & 60.29 \\
Vehicle of transmission to pregnant women & Raw meat & 82.35 \\
& Raw milk & 70.59 \\
& Raw vegetable & 48.53 \\
\hline
\end{tabular}




\begin{tabular}{|c|c|c|}
\hline Questionnaire items & Response category & Respondent $\%(\mathrm{~N}=68)$ \\
\hline \multirow{2}{*}{ Do you screen pregnant women for toxoplasmosis? } & Yes & 8.82 \\
\hline & No & 91.18 \\
\hline \multirow{2}{*}{ Have you ever come across with case of toxoplasmosis in pregnant women? } & Yes & 27.94 \\
\hline & No & 72.06 \\
\hline \multirow{6}{*}{ Symptoms of toxoplasmosis in women? } & Lymphadenopathy & 42.65 \\
\hline & Flu like illness & 76.47 \\
\hline & Encephalitis & 45.59 \\
\hline & Paralysis & 26.47 \\
\hline & Eye problem & 41.17 \\
\hline & Abortion & 69.12 \\
\hline \multirow{3}{*}{ How common is abortion due to toxoplasmosis? } & High & 22.06 \\
\hline & Medium & 48.53 \\
\hline & Low & 29.41 \\
\hline \multirow{3}{*}{ Have you come across with congenital toxoplasmosis in fetus? } & Yes & 10.29 \\
\hline & No & 89.71 \\
\hline & Symptoms & 85.29 \\
\hline \multirow{3}{*}{ Method of diagnosis of toxoplasmosis in fetus } & Serology & 89.71 \\
\hline & Ultrasound & 61.76 \\
\hline & CT scan & 52.94 \\
\hline \multirow{5}{*}{ Clinical symptoms of toxoplasmosis in fetus } & Hydrocephalus & 73.52 \\
\hline & Microcephaly & 29.41 \\
\hline & Retarded brain & 64.70 \\
\hline & Eye problem & 41.18 \\
\hline & Hearing problem & 23.53 \\
\hline \multirow{3}{*}{ Risk of toxoplasmosis to immunocompromised pregnant women? } & High & 77.94 \\
\hline & Medium & 14.71 \\
\hline & Low & 7.35 \\
\hline \multirow{4}{*}{ In which stage of gestation (trimester) is toxoplasmosis highly severe? } & First & 52.94 \\
\hline & Second & 26.47 \\
\hline & Third & 13.24 \\
\hline & Do not know & 7.35 \\
\hline \multirow{6}{*}{ Control and prevention techniques } & Avoiding contact with cat feces & 79.41 \\
\hline & Avoiding stray cats & 76.47 \\
\hline & Avoiding contact with soil & 63.24 \\
\hline & Avoiding raw meat & 86.76 \\
\hline & Avoiding raw milk & 77.94 \\
\hline & Avoiding raw vegetable & 54.41 \\
\hline Do you diagnose toxoplasmosis in your center? & No & 100 \\
\hline \multirow{2}{*}{ Reason for no diagnosis } & No facility & 92.65 \\
\hline & No attention & 89.71 \\
\hline \multirow{3}{*}{ Responsible body to control the disease } & Professionals & 70.59 \\
\hline & Community & 77.94 \\
\hline & Both & 91.18 \\
\hline
\end{tabular}

*: Some of the percentages do not some to $100 \%(\mathrm{~N}=68$, total respondents) because in some cases more than one answer was possible.

\section{Discussion}

Toxoplasmosis is one of the major global zoonotic diseases which have a great impact on human health with more serious outcomes in immunocompromised and pregnant women. The major sources of infection are consumption of contaminated raw meat, water, fruits and vegetables or having close contacts with felines and exposure to soil contaminated with cats' feces. In pregnant women, infection during early pregnancy is more serious compared to infection in late gestation, but the likelihood of disease transmission increases during the progression of pregnancy. Toxoplasma infection in the first trimester often causes abortion and late infection causes premature birth, or may lead to adverse complications and even death in babies $[6,19]$.

This study was designed to assess the knowledge, attitude and practice of the community particularly pregnant women, animal health assistants and medical professionals towards zoonotic importance of toxoplasmosis in Afar region. Knowledge of the community on symptoms, transmission methods, treatment, prevention and control of the disease is not assessed. Moreover, the attitude and current practice towards zoonotic importance of the disease is not studied.

According to the study, out of the 156 pregnant women interviewed only 9 (5.8\%) have ever heard or read about toxoplasmosis and all of those having knowledge about the disease are literate. This finding is in line with Andiappan and other colleagues in 2014 [19] who reported about $11 \%$ of the interviewed pregnant women in Malaysia, Philippines and Thailand had knowledge about the disease in which both findings are by far lower than expected. Since majority of the community in this study area is leading pastoral life, the reason for this low awareness about the disease is illiteracy, no access to medias and no attention is given to the disease in the health centers due to lack of awareness and diagnostic facilities. In this study, 
about $7(77.8 \%)$ of the pregnant women who had knowledge about the disease were from urban area who has access to different media and who got chance to read and hear about the disease from different sources. Moreover, only $12(7.7 \%)$ of the interviewed pregnant women have ever been tested for toxoplasmosis during pregnancy time when they got chance to go out of the region to other cities of the country. However, this disease is among the most important diseases in Ethiopia such as HIV/AIDS, hepatitis $\mathrm{B}$ and tuberculosis that pregnant women should be tested for during pregnancy time.

According to the logistic regression analysis of pregnant women's awareness on zoonotic Toxoplasma infection, literacy was found statistically significant $(\mathrm{P}<0.05)$ in association with awareness of the pregnant women about the disease. This showed that education is critical in increasing attitude of the pregnant women in reading, learning and hearing about the diseases that have importance during pregnancy time. However, the other socio-demographic profiles of the respondents are found statistically not significant.

According to the respondents' answers on the general knowledge of toxoplasmosis, majority of these pregnant women have no knowledge or not sure about this parasitic infection. This finding indicates the importance of educating the pregnant women with the specific preventive measures of the disease which have been discussed in previous studies [20-22, 19].

Most of the respondent pregnant women were not sure about the risk factors, symptoms, and timing of infection of toxoplasmosis. However, they routinely practice primary preventive behaviors, particularly good hygienic condition that can prevent them from Toxoplasma infection but this is due to their native knowledge and thinking that ensuring general and personal hygiene can prevent all types of infection. This is evidenced by most of the pregnant women responded that they wash their hands after gardening $(77.6 \%)$ after changing cat litter $(64.7 \%)$ and after handling raw meat $(62.2 \%)$. Majority of the pregnant women $(84.6 \%)$ responded that they cook meat well before eating however, about $89.7 \%$ of them responded that they drink raw milk and even they consider that drinking raw milk does not transmit Toxoplasma infection. However, there are reports from different parts of the world that there is positive correlation between drinking raw milk and human infection [23-24]. Few other studies investigated the detection of natural infection of the parasite within milk from camel and donkeys in which tachyzoites found in the milk are attributed for transmission of the disease. In Iran, scientists reported a rate of camel milk contamination of $3.12 \%$ by culture bioassays [25].

In addition, about $85.9 \%$ of them responded that they did not avoid drinking untreated water but this is not due to lack of awareness rather due to treated water inaccessibility. Toxoplasma gondii poses a public health problem in both infection rate and disease burden that have been reported in pregnant women from different parts of the world. This parasite has been found in any given environmental conditions that contaminating with cat's feces. There have been reports showing that having a close contact with cats and cleaning their litter may transmit the disease to pregnant women [26]. Exposure to infected cats by these pregnant women could lead to severe outcomes to their carried fetus. Many of these pregnant women knew that by avoiding stray cats $(90.4 \%)$, allowing someone else to change the cat's litter box $(51.3 \%)$, making sure the cat's litter box is changed daily $(63.5 \%)$, cooking meat well $(75.6 \%)$, thoroughly washing and/or peeling all fruits and vegetables before eating $(85.3 \%)$ and cleaning cooking utensil after each use (95.5\%) can prevent all types of diseases but not specifically to toxoplasmosis. According to the finding of this study, literacy play an important role in preventing Toxoplasma infection found in these pregnant women. Residence, marital status, number of children and history of abortion has no statistically significant association with awareness about zoonotic importance of the disease and its preventive measures.

A separate questionnaire was administered to animal health assistants and medical professionals to evaluate the awareness and practices of the professionals on zoonotic Toxoplasma infection. These professionals are also responsible in preventing transmission of this disease if they can work jointly with the community in one health approach. During the study, 24 animal health assistants were participated and almost all had knowledge on zoonotic Toxoplasma infection, transmission, treatment, control and prevention. However, all of them had never diagnosed the disease in animals. This is because that it is difficult for them to diagnose the disease using clinical symptoms only in animals. There is lack of required facility in the veterinary clinic and regional laboratories to diagnose the disease using laboratory analysis. In addition, they had never participated in teaching the community about zoonotic implication of the disease, proper handling and consumption of animal products and the role of raw meat consumption and cat feces in transmission of the disease to pregnant women. This is due to lack of collaborative work between medical and veterinary sectors in awareness creation in the community.

Moreover, out of the 68 medical professionals included in the study, only 23 (33.8\%) had knowledge on zoonotic Toxoplasma infection which is lower than expected when compared with importance of the disease in human health particularly pregnant women and children. Majority of the professionals who had knowledge on the disease knew the transmission, treatment, control and prevention methods correctly. But none of them had ever diagnosed the disease in humans and according to the respondents, the reason for the diagnosis problem was lack of facility $(92.6 \%)$ and no attention was given to the disease $(89.7 \%)$. In addition, most of the medical professionals responded that the major vehicle of transmission for Toxoplasma infection is consumption of raw meat $(82.4 \%)$, and they did not screen pregnant women for the disease $(91.2 \%)$, they had never come across with 
toxoplasmosis case in pregnant women $(72.1 \%)$, and flu like illness $(76.5 \%)$ as a major clinical symptom of the disease. Only $22.1 \%$ of them responded that abortion due to the disease is high, and about $89.7 \%$ of them had never come across with congenital toxoplasmosis in fetus, hydrocephalus $(73.5 \%)$ and retarded brain $(64.7 \%)$ as important symptoms in fetus, and clinical symptom (85.3\%) and serology (89.7\%) as major means of diagnosis of the disease. Moreover, they responded that the risk of toxoplasmosis to immunocompromised pregnant women is high $(77.9 \%)$,the disease is highly severe in first trimester $(52.9 \%)$, avoiding raw meat consumption (86.9) and avoiding contact with cat feces $(79.4 \%)$ as major preventive measures and both the community and the professionals as responsible body to control the disease $(91.2 \%)$.

\section{Conclusion}

According to the study, majority of the pregnant women interviewed had no awareness about zoonotic importance of toxoplasmosis and its preventive measures. According to the respondents' answers on the general knowledge of toxoplasmosis, majority of these pregnant women have no knowledge or not sure about this parasitic infection and were not sure about the risk factors, symptoms, and timing of infection of toxoplasmosis. However, they routinely practice primary preventive behaviors, particularly good hygienic condition that can prevent them from Toxoplasma infection but this is due to their native knowledge and thinking that ensuring general and personal hygiene can prevent all types of infection. According to the finding of this study, literacy play an important role in preventing Toxoplasma infection found in these pregnant women. Almost all animal health assistants participated in the study had knowledge on zoonotic Toxoplasma infection, transmission methods, treatment, control and prevention. However, all of them had never diagnosed the disease in animals. Moreover, most of the medical professionals included in the study had no knowledge on zoonotic Toxoplasma infection in pregnant women and children. However, majority of the professionals who had knowledge on the disease knew the transmission, treatment, control and prevention methods correctly. But none of them had ever diagnosed the disease in humans and according to the respondents, the reason for the diagnosis problem was lack of facility and no attention was given to the disease. Therefore, refreshing training to medical professionals including health extension workers, imparting health education in one health approach to the community at large and pregnant women in particular and strengthening the diagnostic facility and advising pregnant women to regularly check for toxoplasma infection is mandatory.

\section{References}

[1] Dubey, J. P. and Su, C. (2009): Population biology of Toxoplasma gondii: what's out and where did they come from. Mem. Inst. Oswaldo. Cruz., 104:190-195.
[2] Dubey, J. P., Salant, H., Sreekumar, C., Dahal, E., Vinna, M. C., Shen, S. K., Kwok, O. C., Spira, D., Hamburger, J. and Lehman, T. (2004): High prevalence of Toxoplasma gondii in a commercial flock of chickens in Israel, and public health implication of free range farming. Vet. parastol., 121: 317-322.

[3] Carruthers, V. B. (2002): Host cell invasion by the opportunistic pathogen Toxoplasma gondii. Acta. Trop., 81: 111-122.

[4] Beverley, L. K. A, (1974): Some aspects of Toxoplasmosis. A World wide Zoonosis. In: Parasitic Zoonoses. Soulsby Academic Press. New York, Pp 16.

[5] Dubey, J. P. (1994): Toxoplasmosis: Zoonosis Update. JAVMA, 205:1594-1595.

[6] Pereira, K. S., Franco, R. M. and Leal, D. A. (2010): Transmission of toxoplasmosis (Toxoplasma gondii) by foods. Adv. Food Nutr. Res., 60:1-19.

[7] Xiao, Y., Yin, J., Jiang, N., Xiang, M., Hao, L., Lu, H., Sang, H., Liu, X., Xu, H., Ankarklev, J., Lindh, J. and Chen, Q. (2010): Seroepidemiology of human Toxoplasmagondii infection in China. BMC, Infect. Dis., 10:4.

[8] Naoi, K. and Yano, A. (2002): A theoretical analysis of the relation between the risk of congenital toxoplasmosis and the annual infection rates with a convincing argument for better public intervention. Int. Parsitol., 51: 187-194.

[9] Acha, P. N. and Szyfres, B. (2003): Toxoplasmosis. In: Zoonoses and Communicable Diseases Common to Man and Animals. Pan American Health Organization. Washington, D. C. $3^{\text {rd }}$ ed. $P p$ 76-86.

[10] Chen, X. G. and Tan, F. (2009): Toxoplasma gondii: Past, present and future. Records, 27: 426-431.

[11] Sensini, A. (2006): Toxoplasma gondii infection in pregnancy: Opportunities and pitfalls of serological diagnosis. Clin. Microbiol. Infect., 12: 504-512.

[12] Kassali, O. B. and Tekelye, B. (1989): Toxoplasmosis in sheep, goats and cattle in central Ethiopia. Vet. Rese. Commun., 13: 371-375.

[13] Tilaye, D. and Getachew, T. (2002): Study on Toxoplasmosis in sheep and goats in Debre Birhan and surrounding areas in Ethiopia. Bull. Anim. Hlth. Prod. Afr., 50: 138-147.

[14] Teshale, S., Dumetre, A., Darde, M. L., Merga, B. and Dochies, P. (2007): Serological survey of toxoplasmosis in Ethiopia: Prevalence and risk factors. Parasite, 14:155-159.

[15] Negash, T., Tilahun, G. and Medhin, G. (2008): Seroprevalence of Toxoplasma gondii in Nazareth town, Ethiopia. East Afr J Public Health, 5:211-214.

[16] Gebremedhin E. Z., Anteneh H. A., Tesfaye S. T., Kassu D. T., Girmay M., Maria V., Vincenzo D. M., Eric C. and Pierre D. (2013):Sero epidemiology of Toxoplasma gondii infection in women of child-bearing age in central Ethiopia. BMC Infectious Diseases, 13:101.

[17] Hailu, A., Kassahun, N., Aweke, T., Medhint, G., Tesfaye, S., Tariku J. and Daniel F. (2014):Sero-Prevalence and associated risk factors of Toxoplasma gondii infection in pregnant women and HIV/AIDS patients in selected cities ofEthiopia.Banat Journal of Biotechnology, 10:17-29.

[18] CSA (Central Statistical Agency) (2007): Human and animal population census in Afar region. Addis Ababa, Ethiopia. 
[19] Andiappan, H., Veeranoot N., Nongyao S., Si-Lay K., Cristina C. S, Mary M. M.C., Julieta Z. D., Waenurama C., Ching X. T., Yee-Ling L. and Noor A. M. A. (2014): Knowledge and practice on Toxoplasma infection in pregnant women from Malaysia, Philippines, and Thailand. Frontiers in microbiology, 5:291.

[20] Elsheikha, H. M. (2008): Congenital toxoplasmosis: priorities for further health promotion action. Public Health, 122:335-353.

[21] Costa, F. F., Gondim, A. P., Lima, M. B., Braga, J. U., Vieira, L. J. and Araújo, M. A. (2012): Preventive behavior for toxoplasmosis in pregnant adolescents in the state of Ceara, Brazil. BMC Public Health, 12:73.

[22] Amin, T. T., Ali, M. N., Alrashid, A. A., Al-Agnam, A. A. and Al Sultan, A. A. (2013): Toxoplasmosis preventive behavior and related knowledge among Saudi pregnant women. Glob. J. Health Sci., 5:131-143.
[23] Jones, J. L., Dargelas, V., Roberts, J., Press, C., Remington, J. S and Montoya, J. G. (2009): Risk factors for Toxoplasma gondii infection in the United States. Clin Infect Dis., 49: 878-884.

[24] Boughattas, S. (2015): Milkborne toxoplasmosis. Front. Microbiol., 6:215.

[25] Dehkordi, F. S., Borujeni, M. R., Rahimi, E. and Abdizadeh, R. (2013): Detection of Toxoplasma gondii in raw caprine, ovine, buffalo, bovine, and camel milk using cell cultivation, cat bioassay, capture ELISA, and PCR methods in Iran. Food borne Pathog Dis., 10: 120-125.

[26] Dubey, J. P. (2010): Toxoplasmosis of Animals and Humans. $2^{\text {nd }}$ ed. Beltsville, Maryland, U.S.A. CRC Press, Pp $1-338$. 